\title{
Report on the use of communication technology by a sample of public health professionals in NSW
}

\section{Carlie-Jane Naylor ${ }^{\mathrm{A}, \mathrm{D}}$, D. Lynne Madden ${ }^{\mathrm{A}, \mathrm{B}}$ and Deborah J. Oong $\mathrm{C}$}

\author{
A Public Health Training and Development Branch, \\ NSW Department of Health \\ ${ }^{\mathrm{B}}$ Australasian Faculty of Public Health Medicine, \\ Royal Australasian College of Physicians \\ ${ }^{\mathrm{C}}$ Corporate Governance and Risk Management Branch, \\ NSW Department of Health \\ DCorresponding author. Email: carlie.naylor@doh. \\ health.nsw.gov.au
}

\section{Executive summary}

A qualitative survey was carried out with a small sample of senior public health professionals to describe: the types of communication technology that they currently use; the situations in which they apply these at work; and their interest in pursuing these techniques in the future. Six techniques were investigated: teleconferencing, web bulletin boards, web conferencing, videoconferencing, media streaming and satellite television.

Thirteen public health professionals were invited to be interviewed and 12 agreed to participate (response rate $92 \%$ ). Participants were people working in population health structures in the area health services in New South Wales (NSW) and the NSW Department of Health. Participants were interviewed using a structured survey that examined their: roles and responsibilities; experience of using communication technology; and barriers or enablers to their use.

The survey found that two communication techniques, teleconferencing and videoconferencing, are established in practice. Videoconferencing was more likely to be used where personal connection and visual cues were considered to be necessary. Satellite television, like the computer-based techniques, was used by only a third of participants.

Factors that helped participants to engage with communication technology included: ease of access to facilities; assistance provided in organising and setting up the technology; and situations where use of the technology resulted in an efficient and effective use of time and where the use of technology suited the purpose. Participants used what was familiar to them or had been recommended by colleagues. Having knowledge and skills about a particular type of communication technology appeared to lead to its routine application.

In contrast, factors that prevented use were the lack of availability of equipment, the lack of experience and skills in setting up and operating the technology and the perceived associated costs.

Many raised cost as an issue, inferring that cheap delivery increased the chance of uptake. However, there is a tradeoff between cost and quality of delivery for many of these techniques. Better quality transmission of videoconferencing is associated with higher bandwidth transmission and higher cost.

To integrate the use of communication technology into public health practice, a strategy is required that complements the traditional face-to-face approach. The strategy should increase awareness of the types of techniques available, the benefits they provide and the situations in which they are best applied. The challenge for the public health workforce is to clearly articulate their requirements and to successfully and comprehensively integrate a range of communication techniques into practice. 


\section{Background}

Communication technology has an important role in health services in NSW, not only for the delivery of clinical services but also as a means of delivering training, holding meetings and sustaining networks. One reason for this is the large geographical size of NSW; it is divided into eight area health services, the largest of which covers $444586 \mathrm{~km}^{2} .{ }^{1}$ Communication technology provides a means for linking health professionals across areas, particularly those working in rural and remote communities. Where distances are not large, these techniques also allow a more efficient use of time by reducing the need for travel.

Teleconferencing is frequently used for different types of meetings because it is readily available and easy to use. These features led Wildsoet et al. ${ }^{2}$ to pilot a continuing education program via teleconferencing to optometrists in rural Queensland. There are, however, limitations to teleconferencing. A randomised controlled trial compared teleconferencing with videoconferencing for the delivery of multidisciplinary case conferences. ${ }^{3}$ The trial found that there were benefits associated with videoconferencing and, furthermore, a small survey highlighted that having a visual component generated a high level of satisfaction among team members.

Videoconferencing is widely used in medicine for delivering clinical services such as mental health care. ${ }^{4}$ In these clinical settings, there are two sites: one where the clinician is based and a remote site where the patient is located. This type of videoconference is referred to as point-to-point. Further, as we described in a report published in 2002, videoconferencing can be used to deliver continuing professional development activities to multiple remote sites and retain an environment that supports collegiate networking. ${ }^{5}$

Streaming media over the Internet provides a solution when training schedules conflict with other commitments. Chong et al. ${ }^{6}$ described their experience of setting up and delivering presentations by media streaming to pharmacy staff at a Vancouver hospital. As a result of the streamed presentations, staff who were unable to attend the face-toface sessions were able to access the presentations at their own convenience, either on their work or home computer. ${ }^{6}$ Other computer-based applications, such as web conferencing and web bulletin boards, offer alternative ways to support the delivery of training.

Satellite television has also been shown to be an effective medium for delivering continuing education to large groups. The Centers for Disease Control and Prevention (CDC) collaborative has used this medium to deliver courses to multiple sites across the United States. ${ }^{7,8}$

There may be opportunities to expand the contribution of communication technology to public health work. Consequently, to inform strategic planning for the public health workforce and to identify opportunities to use communication technology, a survey was undertaken to provide a 'snapshot' of the use of communication techniques by public health professionals. The forms used, the situations in which these are applied and the level of use were explored.

\section{Method}

Sample

Thirteen public health professionals in NSW were invited via a group email to participate in the survey. To ensure representation across the public health field, a purposeful sample was chosen of at least two people from the population health structures in the area health services, including health promotion and public health. A community health perspective was also sought. Participants were also drawn from the Division of Population Health at the NSW Department of Health. There was rural and metropolitan representation.

\section{Questionnaire}

A structured questionnaire using closed and open-ended questions was designed for the survey. The questionnaire had three parts: the first part sought characteristics of the participants and of their work; the second part asked about their experience in using communication technology and their interest in expanding this; and the third part explored factors that would prevent or assist them to do this. A copy of the survey questionnaire is presented in Appendix 1 .

\section{Part 1: Characteristics of the participants}

Participants were asked about their current role and responsibilities within the NSW Health system, the time required to participate in meetings and continuing professional development activities and time spent travelling to these commitments. Acknowledging the restructuring of health services in NSW into fewer larger area health services, participants were asked whether their role and responsibilities were likely to change and if more travel would be required.

\section{Part 2: Experience of communication technology}

Current use of the following communication techniques was explored: teleconferencing, web bulletin boards, web conferencing, videoconferencing, media streaming and satellite television. To ensure that all the participants had a similar understanding of each technique, a brief description was provided for each. These descriptions were imbedded into the questionnaire and can be viewed in Appendix 1.

\section{Part 3: Barriers and enabling factors}

Factors that would prevent or assist the future use of these techniques were sought, as was the participant's interest in participating in pilots of these techniques.

\section{Preamble}

Before commencing the survey with each participant, the interviewer established the context and the scope of the interview 
using a scripted preamble. The preamble encouraged the participants to consider a range of applications of communication technology within the context of the restructure. A copy of the preamble is included in Appendix 2.

The survey and preamble were piloted with a public health professional and the feedback used to make amendments to the questionnaire and its delivery.

\section{Interviews}

The interviews were scheduled for March and April 2005. The questionnaire was administered to participants by an interviewer either in person or over the telephone. All the interviews were conducted by the one interviewer. Completion of the interviews took between 30 and 40 minutes.

\section{Analysis}

Responses were entered into a database created in Epi Info version 3.3 (CDC, Atlanta, GA). For the closed questions, frequencies were reported. A thematic analysis was carried out on the responses to the open-ended questions.

\section{Results}

\section{Part 1: Characteristics of the participants}

Thirteen public health professionals were invited to be interviewed and 12 agreed to participate. The overall response rate was $92 \%$. The roles and responsibilities of participants spanned: the management of units or teams; leadership of population health and community services; development of evidence-based guidelines and policies; workforce development; facilitating health promotion; and the provision of population health services. The number of people reporting to them varied from one full time equivalent position to approximately 1000, through direct and indirect reporting lines.

Participants reported that between 20 and $80 \%$ of their time was spent in meetings and professional development activities. For eight of the 12 participants most of this time was spent in meetings. Participants reported that the time spent travelling to these activities ranged from 'very little', with meetings being held at their worksite, to approximately 2 hours per day.

Six participants indicated that their role and responsibilities would change as a result of the restructure. Three of these people indicated that the amount of time dedicated to travelling to meet with staff and other organisational functions was increasing. One person reported that their travelling time would decrease and the remaining two reported that this would be dependent on the position they were appointed to within the new structure.

Five indicated that their role and responsibilities would not change as a result of the restructure. Of these, three people indicated that they expected there to be no change in the amount of time spent travelling, while two expected an increase. One person indicated that it was possible that their roles and responsibilities might change as a result of the restructure.

\section{Part 2: Experience of communication technology \\ Teleconferencing}

All participants reported using teleconferencing to either host or participate in meetings. Participants reported using teleconferencing in situations where the distance to travel was prohibitive for a face-to-face meeting or where an urgent issue had to be addressed at short notice. The types of situations where teleconference was employed included: meetings (locally, state-wide and national), interview panels, organising committees, advisory committees and training.

\section{Videoconferencing}

Eleven participants reported using videoconferencing. Videoconferencing was used for a range of activities, including: meetings; research and ethics committees; interviews and selection panels; collaborating on clinical service plans across amalgamated area health services; overseas participants asking questions to a panel at the World Conference on Health Promotion and Health Education in Australia; and other continuing professional development activities. Six respondents cited Bug Breakfast as a professional development activity that was made available to rural and remote sites in NSW by videoconferencing.

One participant reported that videoconferencing had other benefits, including the ability to record a continuing professional development activity and then to circulate this to those who were unable to attend the session.

\section{Web bulletin boards}

Three participants reported using web bulletin boards. One participant cited that Quit Online used a web bulletin board. This was accessible via the NSW Health Intranet and moderated by two health professionals. Two participants reported that within their area health services electronic postings were placed on a noticeboard on the Intranet. In one area health service, staff are alerted to a posting by an email that is generated. Unlike the Quit Online bulletin board, the two other bulletin boards described were limited to posting information and did not facilitate a discussion forum.

When the nine participants who had not previously used web bulletin boards were provided with a brief description, three suggested potential applications to assist in the dissemination of information such as circulars, protocols for statewide networks and occupational health and safety advice for staff.

\section{Web conferencing}

Three participants reported that they had previously used web conferencing; in two cases this was part of a professional 
development activity. Respondents reported using various features of web conferencing, including chat facility, viewing a video and annotating documents.

Eight participants who had not previously used web conferencing indicated that this technique could potentially assist them in their role. They suggested applications such as displaying PowerPoint slides to support presentations delivered via teleconference so that the virtual audience could view the presentation in real time. Respondents highlighted the benefits of the various functions of web conferencing, particularly the ability to share and annotate documents in real time. One respondent suggested sharing applications such as Map Info via web conferencing when seeking assistance from the NSW Department of Health in using this program. Furthermore, web conferencing was suggested as an avenue to demonstrate new software to a widespread public health audience. One person who had not previously used web conferencing was uncertain about how this could be used.

\section{Media streaming}

Three participants reported using media streaming. One person reported accessing archived video streaming from the Internet, for example the Surgeon General's Report from the United States, and locally, being able to access the Environmental Tobacco Smoke and Children commercial from the website.

Four participants who had not used the technology recognised the potential application in the delivery of professional development activities. Three participants were unsure about its application in their work.

\section{Satellite television}

Four participants reported that they had used satellite television. Two described their experiences of using satellite television in other states of Australia: one person had accessed a continuing professional development activity on a regular basis while working in Queensland, and another had viewed media broadcasts and training in Western Australia. One respondent cited the Rural Health Education Foundation as a regular provider of satellite broadcasts on health related topics. One participant reported being interviewed on a live satellite television broadcast.

The area where participants felt that satellite television had the most potential was in the provision of professional development.

\section{Part 3: Barriers and enabling factors Barriers}

Participants cited the following factors as preventing the use of communication technology in their workplace:

- lack of access and availability of equipment
- experience and skills in setting up and operating the technology and

- the associated costs.

Several people highlighted lack of access as a barrier. Reasons cited included: not easily accessible to those who would like to participate, distance travelled to facilities, limited resources (computers) and infrastructure (dial up modems). Participants appreciated that there was a certain level of skill and expertise required to use these techniques successfully. They also reported a limited knowledge of how to use the techniques and that they were unfamiliar with the variety of techniques potentially available and their application.

Participants also emphasised that some areas of public health work do not lend themselves readily to the types of communication technology described in the survey and that face-to-face meetings have an important role to play, for example in liaising with other government departments.

\section{Enablers}

Participants cited the following factors as enabling the use of communication techniques in their work:

- ease of access to facilities

- where assistance is provided in organising and setting up the technology and

- when it resulted in an efficient and effective use of time.

Their application was viewed as potentially cost effective, by providing training opportunities for staff and limiting the need to travel vast distances. Participants acknowledged that communication techniques could lead to positive outcomes in their work by: facilitating interactions with other public health professionals across the state; supporting the achievement of good standards in practice; and increasing access to experts. Furthermore, communication techniques were seen as an effective means of gaining widespread access to people where the distance between them is large.

\section{Opportunities for using communication techniques}

Nine participants indicated that there were opportunities for exploring the use of these techniques in their work and that they were interested in participating in pilots of these. Pilots that they suggested included: the delivery of training, dissemination of information and working collaboratively. The delivery of training was one area that was identified by several respondents where technology could potentially provide a far greater service to the public health workforce. Exploring ways of combining these techniques with methods of face-to-face delivery was proposed. 
There was also a role seen for the utilisation of videoconferencing for public health professionals to attend meetings, particularly in view of the amalgamated area health services, where the distance required to travel across the new larger areas has increased. It was also suggested that following the restructure communication techniques may have a role in maintaining the various public health networks in NSW.

During the interviews participants described using other types of technology that were not raised by the survey. Several reported using list servers. In the former Central Sydney Area Health Service, a pilot was underway where early childhood nurses were using personal digital assistants (PDAs) for collecting information during their home visits. The Quit Smoking Program sends an SMS, or short message service, to the mobile phone of the person who has elected to quit. These messages provide advice and support and their delivery is timed to coincide with when the person can be anticipated to be finding it difficult to sustain quitting.

\section{Discussion}

Two communication techniques, teleconferencing and videoconferencing, appear to be well established in practice. Teleconferencing was the most commonly used; all participants had either participated in or hosted one. Teleconferencing was seen as easy to use and accessible; consequently, it has become integrated into everyday practice. Several participants stated that their telephone had the capacity to connect several lines and that only when it was necessary to connect a large number of people did they use an external service provider. Participants noted that there were skills needed when chairing a teleconferenced meeting, for example that it was important to ensure that everyone had an opportunity to contribute and that this seemed harder than in a face-to-face meeting. This survey did not explore the technical resources available to support good quality teleconferences.

Videoconferencing was the second most commonly used communication technique; only one participant had not used videoconferencing. Like teleconferencing, it was used for conducting meetings, for continuing professional development activities and collaborating with colleagues. Participants liked videoconferencing because it provided a personal connection and visual cues, which were seen as being very important when engagement was required. One participant who described face-to-face meeting as the 'ideal' felt that videoconferencing provided a way to meet in real time when the 'ideal' was not possible.

Satellite television is a modality that generally would be reserved for continuing professional development activities for large groups because it is expensive to provide.
Satellite television is primarily used for the widespread dissemination of information and, consequently, has a potential role in broadcasting continuing professional development activities that need to be delivered to large groups of public health professionals.

The remaining three computer-based modalities had each been used by only a few participants.

This survey was carried out with a small, selected group of public health professionals working in a variety of settings in metropolitan and rural NSW. Several of the public health professionals were drawn from the same area health service and this might have impacted on the findings as it is likely that the types of communication techniques available were similar within the same area health service. Despite this the findings from this survey provide an insight into the current use of these techniques across a broad range of public health practice.

Further, the survey asked about current use and the potential for the future use of these techniques. We did not seek information about difficulties currently experienced with the application of individual techniques, rather a general question was asked about the perceived barriers to their use. Consequently, the framework of the questionnaire may have shaped the type of information that was collected and the very positive response by the participants.

Determining why one particular type of communication technology was used in preference to another was outside the scope of this study. However, in general, participants used what was familiar to them or had been recommended by colleagues. Having knowledge and skills about a particular type of technology appeared to lead to its routine application.

Participants were clear about the factors that help them to engage with these communication techniques: easy access; available assistance; familiarity with the technology; and task-appropriateness. These factors were likely to result in an efficient use of time and positive outcomes from the experience and this in turn encouraged future use.

They were similarly clear about the factors that were a barrier to efficient use: when the equipment is hard to access; technical difficulties and the perception that the modality is unreliable; no support staff to help with either setup or to troubleshoot problems; and low levels of knowledge and skill in the use of these techniques due to lack of training in their operation and application.

An example of how the availability of resources and infrastructure influenced the uptake and ongoing use of 
communication techniques was provided by one participant who stated that in their area of work it was not unusual for several people to share one computer. It could be anticipated that accessing web bulletin boards, web conferencing and media streaming would be more difficult for this group. Although web bulletin boards and archived media streaming sessions can be accessed at a person's own convenience, web conferencing requires the person to schedule a computer at a set time in order to participate. This example demonstrates that when selecting a communication technique for the delivery of a program or meeting it is important to know who has access to the necessary equipment.

It requires effort for people to change the way they do things and participants queried whether it would be worth the extra effort required to learn how to use these techniques. While positive experiences promote use, negative experiences, like poor quality interactions, create disappointment and can quickly discourage people, particularly where they have choice in how a task is managed.

Many raised cost as an issue, inferring that where the delivery was cheap there was a greater chance of uptake. However, there is a trade-off between cost and quality of delivery in many of these techniques. Better quality transmission of videoconferencing is associated with higher bandwidth transmission and this is more expensive. In some instances using lower bandwidths may be a false economy.

Another aspect of cost is the potential to save both the real and opportunity costs associated with travel. One of the major benefits of these techniques was the reduction in travelling time to attend meetings and other functions. Time spent travelling not only has implications in time away from the workplace but also encroaches on time spent with family. Since the amalgamation of the area health services under the restructure of NSW Health, there are fewer larger area health services. This may result in increased travelling time for some people employed in population health services. Just under half of the participants anticipated that it might be necessary for them to travel more. Consequently, there may be potential for communication techniques to have a larger role in assisting public health professionals to meet their local and state obligations.

In many instances, communication technology expands the size of the population that can access activities, making available experiences that would otherwise have restricted access, for example meetings, training, professional development, mentoring and other work-related activities. For many of these individuals, face-to-face attendance is not an option. Consequently, while remote access is highly valued by people it can be a source of additional cost to the system.
Communication technology has the potential to reduce inequities in accessing professional development activities and potentially can sustain and increase the capacity of the rural and metropolitan public health workforce. However, the risk is that inequalities in accessing these techniques will emerge as some groups may have either easier access or greater skill than others in incorporating these techniques into practice as this area continues to develop.

This study demonstrated that participants commonly use teleconferencing and videoconferencing. The challenge is to assist the workforce to be more efficient and effective in their use. For example, given the high level of use of teleconferencing, consideration should be given to ways of ensuring a high quality of interaction such as access to an audio conferencing system. Also, simple guidelines could be developed for participation in teleconferences.

Several participants indicated that communication technologies should not be used to the exclusion of face-toface meetings. It was acknowledged that personal contact contributes to developing rapport and networks. Communication technologies can never completely replace face-toface meetings but provide options when this is not feasible.

\section{Conclusion}

This sample of people working in population health in NSW enthusiastically engaged with considering how different communication techniques might be used to support population health work. They were also able to cite factors that would both aid and hinder uptake. To successfully integrate these techniques into public health practice, a strategy is required that complements the face-to-face approach. The strategy could heighten awareness of the types of communication techniques available, the benefits they offer and the situations in which they are best applied. The strategy could also challenge the public health workforce to articulate their requirements to integrate a range of communication techniques into practice.

\section{Recommendations}

1. That the Population Health Division consider developing a strategy for the population health workforce to utilise communication technology and that it acknowledges that teleconferencing and videoconferencing are commonly used and it promotes their efficient and effective use.

2. That the potential applications of less frequently used communication techniques are explored and promoted where appropriate.

3. That guidelines are developed for the effective use of selected communication techniques.

\section{Acknowledgments}

1. We would like to thank the participants of the survey.

2. A short article from this survey was previously published in the 
Bulletin (Vol. 18(1-2) pp. 13-16). Here we present the full report and the survey questionnaire.

\section{References}

1. Greater Western Area Health Service. 2004/05 Annual Report. Dubbo, NSW: GWAHS Area Office, 2005. Available from: www.gwahs.nsw.gov.au/files/AReport_Web_Version.pdf. (Cited January 2007.)

2. Wildsoet C, Wood J, Parke J. Audio-teleconferencing as a medium for distance learning: its application for continuing education in optometry. Aust J Rural Health 1996; 4(1): 18-27. doi:10.1111/j.1440-1584.1996.tb00182.x

3. Wilson SF, Marks R, Collins N, Warner B, Frick L. Benefits of multidisciplinary case conferencing using audiovisual compared with telephone communication: a randomized controlled trial. J Telemed Telecare 2004; 10(6): 351-4. doi:10.1258/1357633042602026
4. Dossetor DR, Nunn KP, Fairley M, Eggleton D. A child and adolescent psychiatric outreach service for rural New South Wales: a telemedicine pilot study. J Paediatr Child Health 1999; 35(6): 525-9. doi:10.1046/j.1440-1754.1999.00410.x

5. Madden DL, Naylor CJ, Simpson D. An evaluation of videoconferencing Bug Breakfast. N SW Public Health Bull 2003; 14(S-2).

6. Chong E, Balen RM, Jewesson PJ. Delivery of multimedia educational presentations via streaming media. Am J HealthSystem Pharm 2003; 60(23): 2475-8.

7. Maetz HM, Walton W, Smith M, Lincoln R, Galvin M, Tryon C et al. A satellite primer on tuberculosis: a collaboration in distance education. J Public Health Manag Pract 1998; 4(5): 46-55.

8. Umble KE, Cervero RM, Yang B, Atkinson WL. Effects of traditional classroom and distance continuing education: a theory-driven evaluation of a vaccine-preventable diseases course. Am J Public Health 2000; 90(8): 1218-24. 


\section{Appendix 1.}

\section{Survey on the current use of multimedia by the public health workforce}

The first few questions I would like to ask you are about your role and responsibilities within the NSW health system.

1. What is your job title?

2. What is your principle responsibility in this position?

3. How many people are you responsible for?

4. How much time per week do you dedicate towards participating in meetings and professional development activities?

a. How much time would you spend travelling to these?

5. Is it likely that your role and responsibilities will change as a result of the restructure of the health system?

$\begin{array}{ll}\square & \text { Yes } \\ \square \quad \text { No } \\ \square \quad \text { Unsure } \\ \square \quad \text { Other (please comment) }\end{array}$

6. Do you see the amount of time you would be travelling to meet with your staff or other organisational functions as increasing or decreasing as a result of the current restructure?

Increasing

Decreasing

Other (please specify) 
The next set of questions I will be asking are regarding your experiences in using communication techniques, and your interest in pursuing other types of communication techniques in the future. I will provide a brief description of each type of communication technology, in case you are unfamiliar or unclear about what it is.

This question relates to teleconferencing. A teleconference is the interactive exchange of voice of usually more than 2 participants by telephone.

7. Have you ever hosted or participated in a teleconference?

If yes,

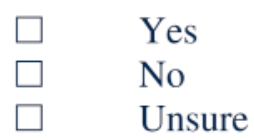

a. Can you please describe the types of situations where you have used teleconferencing?

If no,

b. Can you see how teleconferencing might be applied in your work to assist you in your role?

$\begin{array}{ll}\square & \text { Yes } \\ \square & \text { No } \\ \square & \text { Unsure }\end{array}$

If yes, please explain 
This question relates to web bulletin boards. A web bulletin board is essentially an electronic form of a workplace noticeboard. Messages are posted on an area of a website for other users.

8. Have you ever used a web bulletin board?

Yes

No

Unsure

If yes,

a. Can you please describe the types of situations where you have used a web bulletin board?

If no,

b. Can you see how a web bulletin board might be applied in your work to assist you in your role?

$\begin{array}{ll}\square & \text { Yes } \\ \square & \text { No } \\ \square & \text { Unsure }\end{array}$

If yes, please explain

This question relates to web conferencing. Web conferencing is conducted over the Internet between two or more people situated at different locations. The web conference enables videos, websites, PowerPoint presentations and software demonstrations to be displayed in real time to all the participants through their computer.

9. Have you ever used web conferencing?

$\begin{array}{ll}\square & \text { Yes } \\ \square & \text { No } \\ \square & \text { Unsure }\end{array}$


If yes,

a. Can you please describe the types of situations where you have used web conferencing?

If no,

b. Can you see how web conferencing might be applied in your work to assist you in your role?

$\begin{array}{ll}\square & \text { Yes } \\ \square & \text { No } \\ \square & \text { Unsure }\end{array}$

If yes, please explain

This question relates to videoconferencing. A videoconference is the transmission of video, voice and data between two or more sites.

10. Have you ever used videoconferencing?

Yes

No

Unsure

If yes,

a. Can you please describe the types of situations where you have used videoconferencing? 
If no,

b. Can you see how videoconferencing might be applied in your work to assist you in your role?

$\begin{array}{ll}\square & \text { Yes } \\ \square & \text { No } \\ \square & \text { Unsure }\end{array}$

If yes, please explain

This question relates to media streaming. Streaming media is a method whereby video with synchronised sound is sent in compressed form over the Internet to be viewed as a continuous stream.

11. Have you ever used media streaming, for example video streaming?

$\begin{array}{ll}\square & \text { Yes } \\ \square & \text { No } \\ \square & \text { Unsure }\end{array}$

If yes,

a. Can you please describe the types of situations where you have used media streaming?

If no,

b. Can you see how media streaming might be applied in your work to assist you in your role?

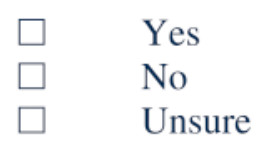

If yes, please explain 
This question relates to satellite television. Satellite television involves a live broadcast over a satellite network.

12. Have you ever used satellite television?

$\begin{array}{ll}\square & \text { Yes } \\ \square & \text { No } \\ \square & \text { Unsure }\end{array}$

If yes,

a. Can you please describe the types of situations where you have used satellite television?

If no,

b. Can you see how satellite television might be applied in your work to assist you in your role?

$\begin{array}{ll}\square & \text { Yes } \\ \square & \text { No } \\ \square & \text { Unsure }\end{array}$

If yes, please explain

The next set of questions relate to factors that would prevent or assist you in using these communication techniques in your work.

13. What prevents you from using these communication techniques in your work? 
14. What helps you to use these communication techniques in your work?

15. Do you see any opportunities in the future for using any of these communication techniques in your work, in addition to what you have already described?

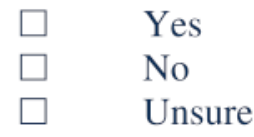

If yes,

Can you provide examples of how you might use these communication techniques in your work?

If yes,

Would you be interested in participating in a pilot of any of these techniques?

Yes

No

Unsure

16. Are there any other comments you would like to make?

Thank you for taking the time to participate in this interview. 


\section{Appendix 2. Preamble to questionnaire}

Thank you for agreeing to be interviewed about your use of communication technologies in your work. The interviews that I am in the process of carrying out will inform a larger piece of work looking at the current communication tools that public health professionals use in their work.

Communication technology is a broad term, but for the purposes of this interview I would like you to reflect on your use of teleconferencing, web bulletin boards, web conferencing, videoconferencing, media streaming and satellite television. I am seeking to obtain a snapshot of how people use these technologies in their day-today work in order to, for example, work with individuals and groups, seek information, and stay in contact with networks relevant to maintain professional knowledge and skills.

When thinking about these technologies in your work please consider meetings, professional development activities, conducting interviews, policy launches and any other areas of your work where these communication technologies may assist you.

We are sampling people in different types of roles and in various locations of NSW. We acknowledge that NSW Health is currently undergoing a restructure and that peoples' roles and responsibilities may be changing. 